\title{
In vitro Growth Characteristics of Fusarium langsethiae Isolates Recovered from Oats and Wheat Grain in the UK
}

\author{
S. M. IMATHIU ${ }^{*}$, R. V. RAY ${ }^{2}$, M. BACK ${ }^{3}$, M. HARE ${ }^{3}$ and S. G. EDWARDS ${ }^{3}$ \\ ${ }^{1}$ Current address: Department of Food Science and Technology, Faculty of Agriculture, \\ Jomo Kenyatta University of Agriculture and Technology, P.O. Box 62000-00100, Nairobi, Kenya \\ ${ }^{2}$ Current address: Division of Crop and Plant Sciences, School of Biosciences, \\ University of Nottingham, Nottingham, United Kingdom \\ ${ }^{3}$ Crop and Environment Sciences Department, Harper Adams University, Newport, United Kingdom
}

(Received: 23 April 2016; accepted: 8 May 2016)

\begin{abstract}
Fusarium langsethiae is a fungus that has recently been implicated in the contamination of small-grain cereal crops such as oats, wheat and barley with high levels of HT-2 and T-2 toxins in many European countries. The epidemiology of this fungus is not well known and may therefore be a bigger problem than currently thought to be. A study was carried out investigating the in vitro growth characteristics of $F$. langsethiae isolates from contaminated oats and wheat at various temperatures; 15, 20, 25 and $30{ }^{\circ} \mathrm{C}$. Results indicated similar growth trends of oats and wheat isolates of $F$. langsethiae. Wheat isolates grew significantly $(\mathrm{p}<0.001)$ faster than oat isolates although this difference may have been confounded by the age of cultures, with oat isolates collected one year earlier. The estimated optimum growth temperature for all isolates was $24{ }^{\circ} \mathrm{C}$. Isolates were macro-morphologically categorized as having lobed or entire colony margins, and either possessing one of the following colony colours: white, orange or purple. Since the estimated optimum growth temperature of F. langsethiae is typical in temperate summers when small-grain cereals are flowering, it is possible that this species can infect, colonise and possibly contaminate the developing grains with HT-2 and T-2 toxins which are of food safety concern.
\end{abstract}

Keywords: Fusarium langsethiae, HT-2 and T-2 toxins, mycotoxins, oats, wheat.

Fusarium langsethiae, whose status as a small-grain cereal crop pathogen has not yet been agreed upon by researchers, is a confirmed toxigenic fungal species known to contaminate oats, wheat and barley with high levels of HT-2 and T-2 trichothecene mycotoxins in Central and Northern Europe (Yli-Mattila, 2010; Edwards et al., 2013; Imathiu et al., 2013). This fungus, which was formerly referred to as 'powdery $F$. poae' was first detailed in the 1990 s as one whose spore morphology resembled that of $F$. poae (Torp and Langseth, 1999) and with a mycotoxin profile similar to that of $F$. sporotrichioides (Thrane et al., 2004). In this regard, it is possible that all T-2 toxin producing strains of $F$. poae reported earlier were actually $F$. langsethiae (Torp and Langseth, 1999). According to Knutsen et al. (2004), due to the similarity of $F$. langsethiae and F. poae micro-

* Corresponding author; e-mail: samuel.imathiu@jkuat.ac.ke 
scopically, the former may often have been identified as the latter. This fungus has been reported to differ from $F$. poae by its slower growth, production of fewer aerial mycelia and presence of powdery appearance on the surface of the colony, and lack of peach-like odour on synthetic media such as potato dextrose agar (Torp and Nirenberg, 2004). On the other hand, F. langsethiae has been shown to differ from F. sporotrichioides in all aspects except the mycotoxin profile and conidial morphology, with the main macroscopic differences between the two species being the rate of colony growth and abundance of aerial mycelia, with $F$. langsethiae growing slower and producing less aerial mycelia in comparison to $F$. sporotrichioides (Thrane et al., 2004).

Due to its ability to colonize and contaminate small-grain cereals with high levels of HT-2 and T-2 toxins, F. langsethiae is considered of great public health concern as mycotoxin contamination of both foodstuff and feedstuff is an unwanted hazard in food and feed chain. These natural microbial toxins have emerged as a significant factor affecting the safety image of cereal grains as a raw material for the food and feed industry (Hietaniemi et al., 2004). T-2 and HT-2 (a deacetylated form of T-2) are type A trichothecenes which are usually found together in contaminated cereal grains and their products. High levels of these mycotoxins have been reported in warm and dry summers in Europe (Langseth and Rundberget, 1999), conditions thought to be conducive for cereal infection and colonization by $F$. langsethiae. HT-2 and T-2 toxins are two of the most potent trichothecenes capable of inhibiting protein synthesis in eukaryotes (Ji et al., 1994; Smith et al., 1994).

As a result of the association of F. langsethiae with HT-2 and T-2 mycotoxin production, this fungal species has recently received great research attention. The natural occurrence of T-2 toxin has been of particular interest in Europe because of the association of T-2 toxin-producing Fusarium species, which caused an outbreak of alimentary toxic aleukia (ATA) that was responsible for the death of thousands of people in Russia during World War II in the 1940s (Desjardins, 2006). However, there is not sufficient information available with regard to the conditions under which $F$. langsethiae grows both in vitro and in vivo while producing these toxins in cereal grains during crop growth and/or storage. Although the European Commission (EC) has established a group tolerable daily intake of $100 \mathrm{ng} \mathrm{kg}^{-1}$ body weight for the sum of HT-2 and T- 2 toxins, the Commission has recommended collection of more data on these toxins on cereals and cereal products including information about conditions contributing to their presence in these commodities, which is currently limited (EC, 2013).

Fusarium species are normally field fungi whose extent of infection, colonization and mycotoxin production is heavily influenced by environmental factors such as temperature and humidity that cannot be controlled under natural conditions (Barbara et al., 2004). For this reason, complete elimination from agricultural commodities is unachievable. A key goal in the production of safe food and feed is, therefore, to develop and/ or adopt practices that reduce the extent of contamination by these toxins to levels that are considered to have no significant impact on health (Desjardins, 2006). This cannot be achieved without an understanding of specific conditions that directly influence the growth of a particular fungus. It is important to understand the growth characteristics of an organism in order to devise ways of reducing mycotoxin contamination. 
It is not known whether $F$. langsethiae is part of the fusarium head blight (FHB) complex of organisms whose growth characteristics and conditions in which they thrive optimally are well known and documented. Temperature is the single most important abiotic factor influencing all aspects of behaviour of every living organism, small changes of which may greatly influence the incidence and severity of infection in small-grain cereals (Brennan et al., 2005). The aims of this study were: to isolate and characterize $F$. langsethiae isolates from commercial UK oats and wheat grains, and to investigate the effect of temperature on the in vitro growth rate of oats and wheat isolates of $F$. langsethiae.

\section{Materials and Methods}

\section{Isolation of $\mathrm{F}$. langsethiae from oat and wheat grains}

Oats and wheat grain samples from years 2001, 2002 and 2004 UK harvests with known low and high levels of HT-2 and T-2 mycotoxins were identified from previous project material (Edwards, 2007a; 2007b). Fusarium langsethiae was isolated from two oat samples with $>500 \mu \mathrm{g} \mathrm{kg}^{-1}$, four oat samples with $<10 \mu \mathrm{g} \mathrm{kg}^{-1}$ and seven wheat samples with $>50 \mu \mathrm{g} \mathrm{kg}^{-1}$ HT-2 and T-2 toxins. Grain samples were thoroughly mixed before representative subsamples were taken. Grains (approximately $20 \mathrm{~g}$ ) were scooped into $50 \mathrm{ml}$ sterile universal tubes. They were soaked in sodium hypochlorite (1.2\% available chlorine) with added Tween $20(0.05 \%)$ for 3 min to surface-sterilize. They were then rinsed three times with sterile distilled water before being allowed to dry in Petri dishes in a laminar air flow cabinet. Grains were plated (5 grains per plate) on potato dextrose agar (PDA, Merck, Germany) amended with streptomycin sulphate $\left(130 \mu \mathrm{g} \mathrm{ml}^{-1}\right)$ to inhibit bacterial growth and incubated at room temperature (ca. $22{ }^{\circ} \mathrm{C}$ ) for 7 days. Fusarium langsethiae was identified by micro- and macro-morphology (Torp and Langseth, 1999; Torp and Nirenberg, 2004). The identity of the fungal isolates was confirmed by real-time PCR as described in Edwards et al. (2013) using the fungal isolates DNA extracted using a rapid DNA extraction method modified from Walsh et al. (1991). Mycelia of the fungal isolates were removed from the surface of PDA plate cultures using a sterilized scalpel and placed in 2.0-ml Eppendorf tubes into which $250 \mu \mathrm{l}$ chelex carbon buffer $(1 \mathrm{~g}$ chelex 100 and $0.25 \mathrm{~g}$ charcoal (granular 20-60 mesh activated) made up to $20 \mathrm{ml}$ using distilled water) was added. Mycelia were crushed with a sterile micro-pestle and incubated at 56 ${ }^{\circ} \mathrm{C}$ for $20 \mathrm{~min}$, vortexed and left to cool at room temperature for $30 \mathrm{~min}$. The tube content was vortexed and centrifuged at $12,000 \times g$ for 15 min after which $50 \mu$ l supernatant was removed and added to $50 \mu \mathrm{TE}$ buffer (10 mM Tris-HCl, $1 \mathrm{Mm}$ EDTA, pH 8.0) and vortexed. Extracted DNA was stored at $4{ }^{\circ} \mathrm{C}$ and $5 \mu \mathrm{l}$ used directly for PCR reactions.

To confirm the identity of the isolates previously identified by micro- and macro-morphology, DNA from the fungal isolate was subjected to $F$. langsethiae real-time PCR. An iCycler PCR machine (Bio-Rad, UK) was used for the amplification of fungal DNA extractions. F. langsethiae DNA standards (100-10-4 ng/ $\mu$ l) were included in duplicate, as well as negative and positive controls. The amplification mix consisted of $1 \mu \mathrm{M}$ F. langsethiae primer pairs (FlangF3 5'-CAAAGTTCAGGGCGAAAACT and LanspoR1 
5'-TACAAGAAGACGTGGCGATAT) (Wilson et al., 2004) and SYBR Green Jumpstart Taq ReadyMix (Sigma, USA) reagent which was used according to manufacturer's instructions. The volume of DNA sample in the reactions was $5 \mu \mathrm{l}$ in a total volume of $25 \mu \mathrm{l}$. In the negative control, $5 \mu \mathrm{l}$ of PCR-grade water was used instead of DNA sample. The PCR programme had an initial denaturation for $2 \mathrm{~min}$ at $95^{\circ} \mathrm{C}$ followed by 40 cycles with $15 \mathrm{~s}$ at $95{ }^{\circ} \mathrm{C}$ (denaturation), $10 \mathrm{~s}$ at $62{ }^{\circ} \mathrm{C}$ (annealing), $30 \mathrm{~s}$ at $72{ }^{\circ} \mathrm{C}$ (extension) and $10 \mathrm{~s}$ at $82^{\circ} \mathrm{C}$ (fluorescence measurement).

In vitro growth study

A single isolate was maintained from each grain sample unless multiple isolates with distinct morphology colour were present. This reduced the chance of having multiples of the same isolate within the study. Ten F. langsethiae isolates (Table I) each from oats and wheat grains were randomly selected for the study. To obtain single spore colonies, each isolate was cultured on PDA as described above until profuse sporulation (pow-

\section{Table 1}

Oat and wheat Fusarium langsethiae isolates used in the in vitro growth rate study

\begin{tabular}{|c|c|}
\hline Isolate & Host cereal \\
\hline Fl 2004/59 & Oat \\
\hline Fl 026/1 & Oat \\
\hline $\mathrm{Fl} 04 / \mathrm{O} 2$ & Oat \\
\hline $\mathrm{Fl} \mathrm{04/O3}$ & Oat \\
\hline Fl 2004/37/1 & Oat \\
\hline Fl 2004/37/2 & Oat \\
\hline Fl 077/3 & Oat \\
\hline Fl 2004/31 & Oat \\
\hline Fl 062/1 & Oat \\
\hline Fl 062/2 & Oat \\
\hline W2004/171a & Wheat \\
\hline W2004/140a & Wheat \\
\hline W2001/69a & Wheat \\
\hline W2004/171b & Wheat \\
\hline W2001/69b & Wheat \\
\hline W2001/17 & Wheat \\
\hline W2004/140 & Wheat \\
\hline W2001/1 & Wheat \\
\hline W2004/170 & Wheat \\
\hline W2004/171c & Wheat \\
\hline
\end{tabular}


dery appearance) had occurred. Spores were carefully scrapped with a sterile wire loop and streaked on water agar (20 g of agar in 11 of tap water) in Petri dishes and incubated at room temperature (ca. $22{ }^{\circ} \mathrm{C}$ ) for 3 days. A marker pen was used to highlight a single distinct colony from the reverse side of the plate which was then, by means of a sterile scalpel transferred onto a fresh PDA plate.

Five millimetre discs of single spore colonies from 12-day-old cultures were cut with a sterile cork borer from an advancing margin of colonies and plated at the centre of four fresh PDA plates. The plates were incubated at $15,20,25$ or $30{ }^{\circ} \mathrm{C}$ in an incubator (Labheat Incubator, UK) in complete darkness for 12 days. Growth was measured by taking two diametric measurements of the colonies perpendicular to each other. The growth of cultures was measured after 3, 6 and 9 days of incubation and growth rate calculated as $\mathrm{mm}$ day $^{-1}$. Other secondary characteristics monitored were colony colour on days 6 and 12 after incubation, colony margin shape on days 6 and 9 and sporulation on days 3, 6, 9 and 12. Experiments were set up in randomized block designs with four replicates. The growth rate data was subjected to analysis of variance (ANOVA) using GenStat (Release 8.1, Rothamsted Experimental Station, UK).

\section{Results}

\section{Isolation of $\mathrm{F}$. langsethiae from the grain}

From oat and wheat samples containing $>500$ and $50 \mu \mathrm{g} \mathrm{kg}^{-1}$ HT-2 and T-2 toxins, respectively, F. langsethiae was isolated from 5-10\% of the grain. No F. langsethiae was isolated from oat samples containing $<10 \mu \mathrm{g} \mathrm{kg}^{-1} \mathrm{HT}-2$ and T-2 toxins. Fusarium langsethiae was identified micro-morphologically (globose to napiform conidia) and macro-morphologically (profuse 'powdery' appearance on short aerial mycelia on agar surface (Fig. 2)). The identity of the isolates was confirmed by real-time PCR methodology detailed by Edwards et al. (2013). The greatest challenge encountered in the isolation process was the fact that $F$. langsethiae was readily overgrown and outcompeted by faster growing fungi on the agar making it difficult to clearly see and effectively and efficiently isolate the fungus.

\section{Effect of temperature on the in vitro growth rate of oats} and wheat isolates of $\mathrm{F}$. langsethiae

The growth trends of isolates of $F$. langsethiae recovered from oats and wheat were found to be similar at the four temperatures of study: 15, 20, 25 and $30{ }^{\circ} \mathrm{C}$ (Fig. 1). All isolates grew least at the most extreme temperatures of 15 and $30^{\circ} \mathrm{C}$ with higher rates of growth observed between 20 and $28^{\circ} \mathrm{C}$. The optimum growth temperature for all isolates was estimated to be about $24^{\circ} \mathrm{C}$. Although the wheat and oats isolates had similar growth trends at all temperatures studied, on average wheat isolates grew faster than the oat isolates at each temperature. Wheat isolates grew significantly faster $(p<0.05)$ than oat isolates at 20,25 and $30{ }^{\circ} \mathrm{C}$ on day 3 . On days 6 and 9 , wheat isolates grew significantly 


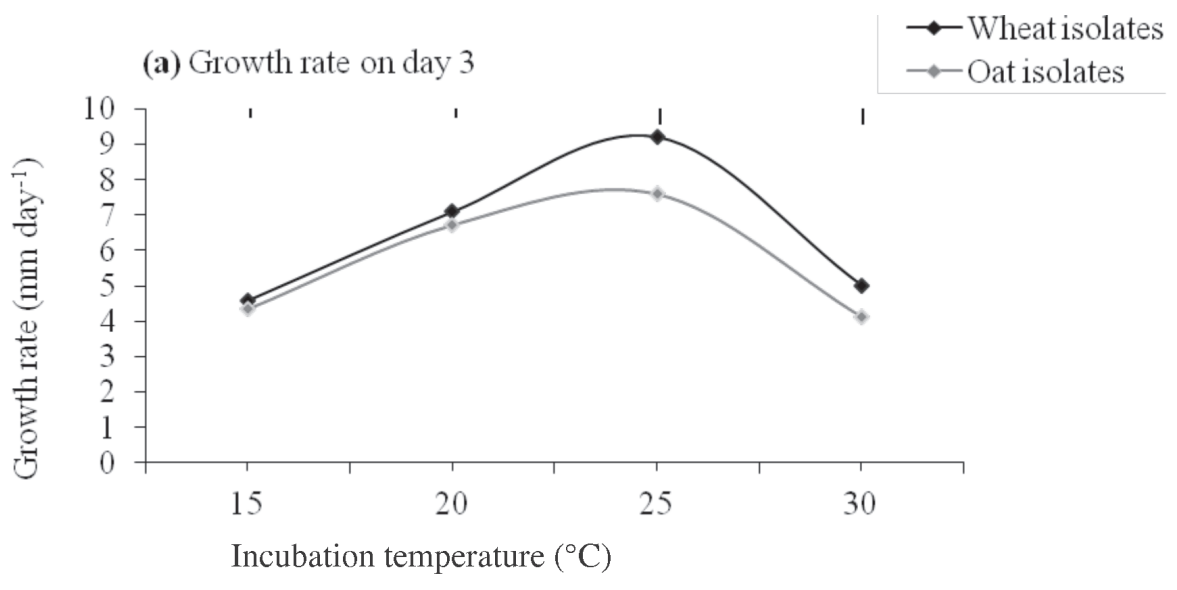

(b) Growth rate on day 6

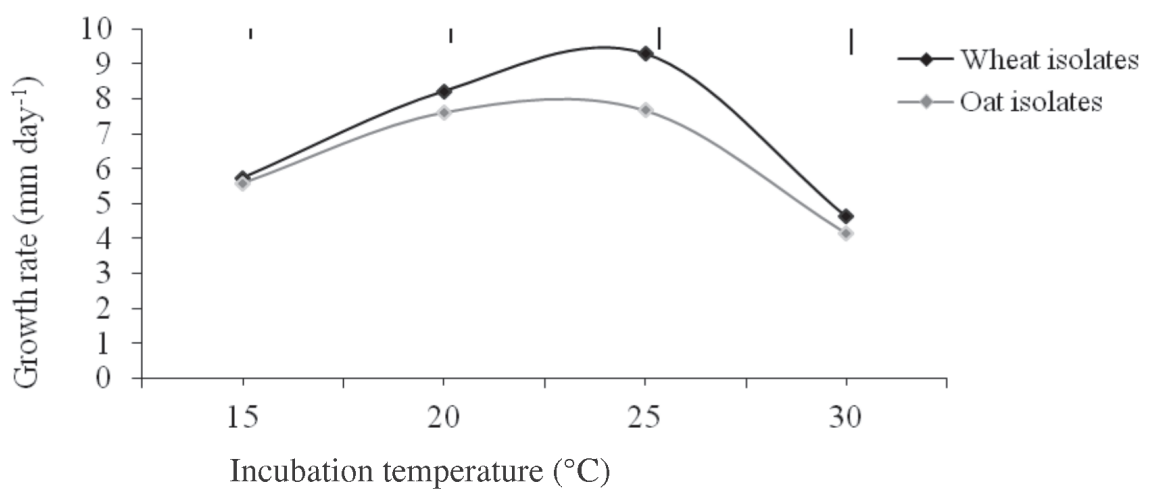

(c) Growth rate on day 9

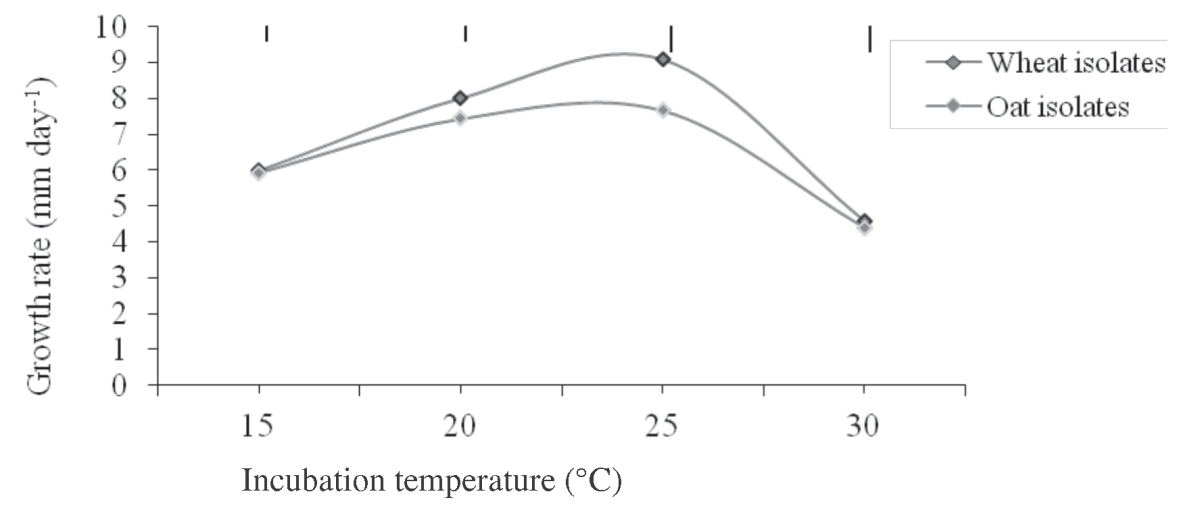

Fig. 1. Effect of temperature on the growth rate of $F$. langsethiae isolates obtained from oat and wheat grains contaminated with HT-2 and T-2 toxins. Values are the means of four replicates. Bars represent least significant difference (LSD (5\%)) 
faster $(p<0.05)$ than oat isolates at 20 and $25^{\circ} \mathrm{C}$ with no significant growth differences at 15 and $30{ }^{\circ} \mathrm{C}(p>0.05)$. The mean growth rates of oat and wheat isolates at $25^{\circ} \mathrm{C}$, a temperature which is close to the estimated optimum temperature for all isolates $\left(24^{\circ} \mathrm{C}\right)$ were as follows: 7.6, 7.7 and $7.7 \mathrm{~mm} \mathrm{day}^{-1}$ for oat isolates on days 3, 6 and 9, respectively; 9.2, 9.3 and $9.1 \mathrm{~mm} \mathrm{day}^{-1}$ for wheat isolates on days 3, 6 and 9, respectively. On day 6 which was considered the optimal day post-plating out for taking growth rate readings, oat isolate Fl 2004/59 had the slowest growth rate while wheat isolate W2001/69(b) had the fastest growth rate recorded over the four incubation temperatures.

\section{Fusarium langsethiae isolates colony morphological characteristics}

In all isolates, colony margins were observed to be either entire or lobed (Fig. 2). The entire colony margin was characterised by smooth advancing colony edges while a lobed margin was characterised by uneven advancing colony edges. Colony mycelial colour of isolates was either white, orange or purple, which also applied to the reverse side of the plates. Only one isolate was purple in colour (isolate Fl 2004/59) (Fig. 2) which was isolated from oats and which had the slowest growth rate over the four incubation temperatures. Pigmentation tended to be more intense with increasing incubation temperature and was more pronounced at $25^{\circ} \mathrm{C}$ than at any other temperature. There was no relationship between colony colour and margin shape observed.

All isolate colonies exhibited a 'powdery' appearance with short sparse mycelia on the agar surface (Fig. 2). Onset and degree of sporulation (profuse 'powdery' appearance on agar surface) depended mainly on incubation temperature. It was observed that the higher the incubation temperature, the earlier was the onset of sporulation. Although very little growth occurred at $30{ }^{\circ} \mathrm{C}$ for all isolates, abundant sporulation was observed on the small colonies. The degree of sporulation was also found to increase with the increase in incubation period, with greatest sporulation observed at $25^{\circ} \mathrm{C}$ on day 9 . Of all the days that the readings were taken, it was evident that macromorphological characteristics for all the isolates were well defined on day 6.

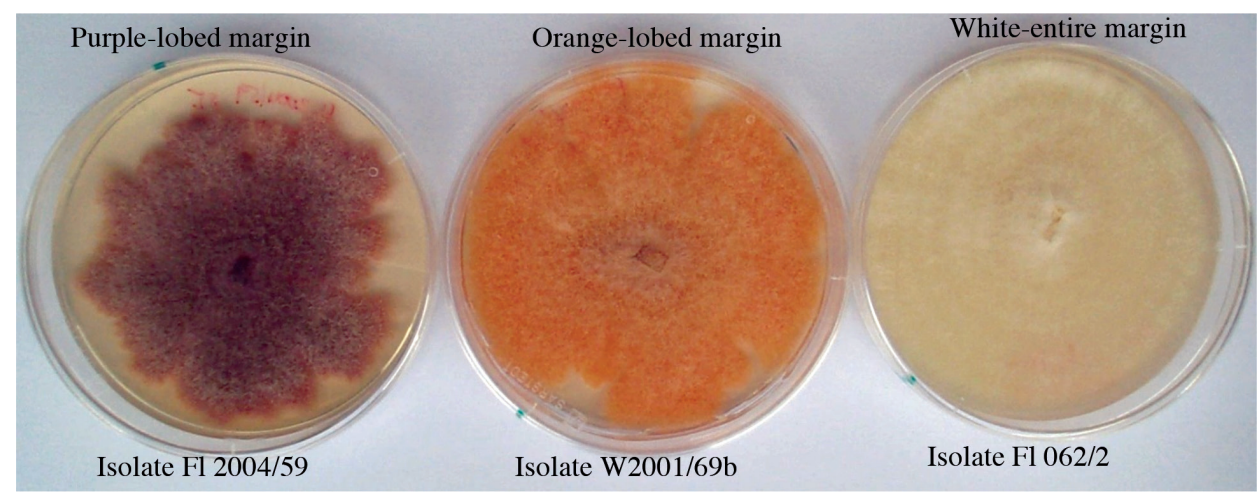

Fig. 2. Three categories of F. langsethiae isolates based on colony colour 


\section{Discussion}

The finding that $F$. langsethiae isolation was problematic as it tended to be overgrown by other types of fungi was not an unique observation. This finding agrees with that reported earlier by Torp and Nirenberg (2004) that, due to its slow growth rate and sparse aerial mycelia, $F$. langsethiae is readily overtaken by other quick growing fungi such as $F$. poae and F. culmorum. This may also explain why low numbers (5-10\%) of the fungus was recovered even from grain samples that were highly contaminated by HT- 2 and T-2 toxins and assumed to be heavily infected.

Generally, most fungi particularly phytopathogenic ones are known to be mesophilic, growing well between $20-30{ }^{\circ} \mathrm{C}$. Most Fusarium species have also been found to grow well between these two temperatures (Smith et al., 1984). Fusarium langsethiae is therefore a mesophile and shares the same optimal growth temperature range with most Fusarium species. Most Fusarium species pathogenic to oats and wheat such as F. poae, $F$. culmorum and $F$. graminearum are known to have an optimum growth temperature of $25^{\circ} \mathrm{C}$ and an optimal growth rate in the range of $20-30{ }^{\circ} \mathrm{C}$ (Brennan et al., 2003; Doohan et al., 2003; Hope et al., 2005). For instance, in vitro growth rates of $F$. culmorum and $F$. graminearum were found to increase between 10 and $25^{\circ} \mathrm{C}$ and decrease between 25 and $30{ }^{\circ} \mathrm{C}$; optimal growth occurred at $25^{\circ} \mathrm{C}$ for $F$. graminearum and $20-25{ }^{\circ} \mathrm{C}$ for F. culmorum. However, a study carried out by Torp and Nirenberg (2004) indicated that the Norwegian isolates of $F$. langsethiae had an optimum growth rate at $27.5^{\circ} \mathrm{C}$. Possible explanations for this difference may be attributed to regional differences including the environment from which the authors' isolates were recovered and the media conditions under which the isolates were grown. Brennan et al. (2003), in a study on the in vitro growth and pathogenicity of European Fusarium fungi showed different growth rates for different Fusarium species from different countries.

Although the oat and wheat isolates of $F$. langsethiae had similar growth trends at all temperatures studied, on average wheat isolates grew faster than the oat isolates at each temperature. It is possible that the significant differences in growth rate observed between oat and wheat isolates may be confounded by the original age of cultures used; oat isolate cultures were older than wheat isolates by one year, while wheat isolates were freshly used in the study after isolation. Hallsworth and Magan (1996) reported that increased age of the culture can contribute to the decline of fungal spore viability. The authors were investigating the effects of culture age, temperature and $\mathrm{pH}$ on the polyol and tetrahalose contents of fungal propagules. Although they did not comment on the growth rates of fungal colonies, age may also influence the rate of growth as observed in the study.

Both colony micro- and macromorphological characteristics which include colony diameter, colony colour and colony margin characteristics have been used in Fusarium taxonomy for a long time and have been known to provide useful secondary criteria for distinguishing isolates (Burgess et al., 1988; Summerell et al., 2003). For many Fusarium species, these characteristics are the only ones that are well described and widely available, and therefore they are very important in distinguishing isolates compared with biological species information and DNA sequence information which are recent advances and not readily available (Summerell et al., 2003). 
A number of colony macroscopic attributes; mycelial colour, colony margin shape and sporulation were assessed in order to characterise the isolates of $F$. langsethiae. Colony pigmentation is known to be uniform within some Fusarium species while in others, it is a variable character (Burgess et al., 1988). In this study, F. langsethiae appeared to be one of those species that may naturally produce different colour shades irrespective of whether isolates are recovered from oats or wheat grains. The different shades of colour in F. langsethiae cultures can be explained by the fact that some of the strains of this fungus have been reported to produce a pigment called aurofusarin (Thrane et al., 2004), which is produced by nearly all strains of $F$. poae and $F$. sporotrichioides and influences colony colour development. Fusarium poae and F. sporotrichioides have been reported to be closely related to $F$. langsethiae micro-morphologically and this might be another extra character they have in common. Similar results on $F$. langsethiae pigmentation were observed by Torp and Nirenberg (2004) who used words like white to pinkish white, pale red or pastel red and orange tint to describe colony pigmentation of this fungus. There is also a possibility that different shades of colour may result because the isolates in the three groups (white, orange and purple) are different subgroups within the same species, which warrants further studies. Colony margins for all the isolates were observed to be either entire or lobed. This was an inconsistent attribute as it could differ in the four replicates of the same isolate at the same incubation temperature indicating that although this secondary attribute can be used to aid isolate identity in some fungi, it is of no use in the characterisation of $F$. lang sethiae isolates.

'Powdery' appearance on the agar surface which is as a result of profuse production of spores is an attribute which is obvious with the unaided eye and taxonomically one of the characters used to differentiate F. langsethiae from all other Fusarium species (Torp and Langseth, 1999; Torp and Nirenberg, 2004). UK Fusarium langsethiae isolates used in this study seem to share all of the growth characteristics reported for European isolates used in a study by Torp and Nirenberg (2004).

Day 6 post-plating out would be considered the most appropriate time for taking all readings on growth rate, pigmentation and other macroscopic characters as at this day, all isolates had grown substantially with all attributes well developed. Day 3 would not be appropriate because all the characters were not well defined. By day 9, a few isolate colonies had grown to reach the edge of the agar plates $\left(20\right.$ and $25^{\circ} \mathrm{C}$ only), which would make assessment of colony margins difficult. The fact that most readings on Fusarium species are taken on days 6 and 7 when cultured on synthetic media (Smith et al., 1984) supports the above statement.

In conclusion, this study has found out that oats and wheat isolates of $F$. langsethiae can grow and sporulate at a wide range of temperatures from $15-30{ }^{\circ} \mathrm{C}$ (and probably lower or higher temperatures at a slower rate). With the optimum growth temperature of F. langsethiae being about $24{ }^{\circ} \mathrm{C}$, it is possible therefore that this species of fungus can infect and colonise small-grain cereals at these temperatures that are common in temperate summers when crops are flowering. The differences in colony colour observed in F. langsethiae isolates may indicate underlying differences in genetic makeup. It would, therefore, be useful to carry out DNA profiling to establish whether isolates differing in colony colour, as well as oat and wheat isolates are distinct subgroups of F. langsethiae. 


\section{Acknowledgements}

Authors wish to thank Quaker Oats, Home-Grown Cereals Authority and Harper Adams University for funding this study.

\section{Literature}

Barbara, K., Mona, T., Eystein, S. and Birgitte, A. (2004): Alternaria and Fusarium in Norwegian grains of reduced quality-a matched pair sample study. Int. J. Food Microbiol. 93, 51-62.

Brennan, J. M., Fagan, B., van Maanen, A., Cooke, B. M. and Doohan, F. M. (2003): Studies on in vitro growth and pathogenicity of European Fusarium fungi. Eur. J. Plant Pathol. 109, 577-587.

Brennan, J. M., Egan, D., Cooke, B. M. and Doohan, F. M. (2005): Effect of temperature on head blight of wheat caused by Fusarium culmorum and F. graminearum. Plant Pathol. 54, 156-160.

Burgess, L. W., Liddell, C. M. and Summerell, B. A. (1988): Laboratory Manual for Fusarium Research, $2^{\text {nd }}$ ed. Department of Plant Pathology and Agricultural Entomology, University of Sydney, Australia.

Desjardins, A. E. (2006): Fusarium Mycotoxin Chemistry: Genetics and Biology. American Phytopathological Society. St. Paul, Minnesota, pp. 72-98.

Doohan, F. M., Brennan, J. and Cooke, B. M. (2003): Influence of climatic factors on Fusarium species pathogenic to cereals. J. Plant Pathol. 109, 755-768.

European Commission. (2013): Commission recommendation of 27 March 2013 on the presence of T-2 and HT-2 toxins in cereals and cereal products. Official Journal of European Union. Available from https:// www.fsai.ie/uploadedFiles/Recomm2013_165.pdf, accessed on $10^{\text {th }}$ August, 2015.

Edwards, S. G. (2007a): Investigation of Fusarium mycotoxins in UK barley and oat production. HGCA Project Report No. 415. HGCA, London.

Edwards, S. G. (2007b): Investigation of Fusarium mycotoxins in UK wheat production. HGCA report number 413.

Edwards, S. G., Imathiu, S. M., Ray, R. V., Back, M. and Hare, M. C. (2013): Molecular studies to identify the Fusarium species responsible for HT-2 and T-2 mycotoxins in UK oats. Int. J. Food Microbiol. 156, $168-175$.

Hallsworth, J. E. and Magan, N. (1996): Culture age, temperature and pH affect the polyol and trehalose contents of fungal propagules. Appl. Environ. Microbiol. 62, 2435-2442.

Hietaniemi, V., Kontturi, M., Eurola, M., Ramo, S., Niskanen, M., Kangas, M. and Saastamoinen, M. (2004): Content of trichothecenes in oats during official variety, organic cultivation and nitrogen fertilization trials in Finland. Agric. Food Sci. 13, 37-54.

Hope, R., Aldred, D. and Magan, N. (2005): Comparison of environmental profiles for growth and deoxynivalenol production by Fusarium culmorum and F. graminearum on wheat grains. Lett Appl. Microbiol. 40, 295-300.

Imathiu, S. M., Edwards, S. G., Ray, R. V. and Back, M. (2013): Fusarium langsethiae - a HT-2 and T-2 toxins producer that needs more attention. J. Phytopathol. 161, 1-10.

Ji, X., Ning, K., Liang, D. and Shi, G. (1994): Effects of sterigmatocystin and T-2 toxin on the induction of unscheduled DNA synthesis in primary cultures of human gastric epithelial cells. Nat toxins 2, 115-119.

Knutsen, A. K., Torp, M. and Holst-Jensen, A. (2004): Phylogenetic analyses of the Fusarium poae, Fusarium sporotrichioides and Fusarium langsethiae species complex based on partial sequences of the translation of elongation factor-1 alpha gene. Int. J. Food Microbiol. 95, 287-295.

Langseth, W. and Rundberget, T. (1999): The occurrence of HT-2 toxin and other trichothecenes in Norwegian cereals. Mycopathologia 147, 157-165.

Smith, J. E., Mitchell, I. and Chiu, L. C. (1984): The natural occurrence of Fusarium mycotoxins. In: M. O. Moss and J. E. Smith (eds): The Applied Mycology of Fusarium. Cambridge University Press, Cambridge, pp. 157-173.

Smith, J. E., Lewis, C. W., Anderson, J. G. and Solomons, G. L. (1994): Mycotoxins in Human Nutrition and Health. EEC, Luxembourg, pp. 201-220. 
Summerell, B. A., Sallech, B. and Leslie, J. F. (2003): A utilitarian approach to Fusarium identification. Plant Dis. 87, 117-128.

Thrane, U., Adler, A., Clasen, P., Galvano, F., Langseth, W., Lew, H., Logrieco, A., Nielen, K. F. and Ritieni, A. (2004): Diversity in metabolite production by Fusarium langsethiae, Fusarium poae and Fusarium sporotrichioides. Int. J. Food Microbiol. 95, 257-266.

Torp, M. and Langseth, W. (1999): Production of T-2 toxin by a Fusarium resembling Fusarium poae. Mycopathologia 147, 89-96.

Torp, M. and Nirenberg. H. I. (2004): Fusarium langsethiae sp. nov. on cereals in Europe. Int. J. Food Microbiol. 95, 247-256.

Walsh, P. S., Metzger, D. A. and Higuchi, R. (1991): Chelex-100 as a medium for simple extraction of DNA for PCR-based typing from forensic material. BioTechniques 10, 506-513.

Wilson, A., Simpson, D., Chandler, E., Jennings, P. and Nicholson, P. (2004): Development of PCR assays for the detection and differentiation of Fusarium sporotrichioides and Fusarium langsethiae. FEMS Microbiol. Lett. 233, 69-76.

Yli-Mattila, T. (2010): Ecology and evolution of toxigenic Fusarium species in cereals in northern Europe and Asia. J. Plant Pathol. 92, 7-18. 